\title{
COX-2 expression during early lung squamous cell carcinoma oncogenesis
}

\author{
C. Mascaux*,+, B. Martin*, J-M. Verdebout" ${ }^{\#}$, V. Ninane ${ }^{\star}$ and J-P. Sculier*
}

ABSTRACT: Cyclooxygenase (COX)-2 is implicated in the oncogenesis of many cancers, and COX-2 inhibitors are effective in preventing the development of tumours, such as in colon cancer. Its expression is increased in nonsmall cell lung cancer and is associated with poor prognosis. The present study assessed COX-2 expression in normal bronchial epithelium, as well as in all the putative precursors of squamous cell carcinomas.

COX-2 expression was studied by immunohistochemistry in 106 biopsies collected during autofluorescence bronchoscopy in consecutive patients at high-risk for lung cancer.

All biopsies corresponding to normal epithelium or low-grade lesions (lesions up to moderate dysplasia) did not show increased COX-2 expression. Lesions were positive for COX-2 in eight out of 14 severe dysplasia patients, eight out of 14 in situ carcinomas and five out of eight invasive carcinomas. A strong statistically significant difference in COX-2 expression was found between normal epithelium or low-grade lesions and high-grade lesions (severe dysplasia or worse). The positive and negative predictive values of COX-2 expression for high-grade lesion were $100 \%$ and

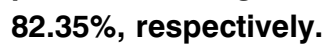

In conclusion, bronchial precursors of squamous cell carcinoma showed increased cyclooxygenase-2 expression and were segregated into low- versus high-grade with a high positive predictive value. Thus, cyclooxygenase-2 appears as a potential early marker of squamous cell carcinoma.

KEYWORDS: Carcinogenesis, chemoprevention, cyclooxygenase-2, lung cancer, pre-malignant lesion, tumour markers

ung cancer is the most common cause of cancer deaths in the Western world, and accounts for more deaths than prostate, breast and colorectal cancers combined [1]. The prognosis for nonsmall cell lung cancer (NSCLC) has not improved significantly, with a 5-yr survival rate still of $<10 \%$. This poor prognosis for lung cancer is mainly due to the diagnosis of a large majority of NSCLC at locally advanced or metastatic stages combined with a lack of effective, innovative and targeted treatment protocols. Understanding the molecular mechanisms that underlie the pathogenesis of lung cancer should open up new opportunities for the development of innovative NSCLC therapies. Recently, attention has focused on the role of prostaglandins and cyclooxygenase (COX) in cancer after the discovery that colonic polyps in patients with familial adenomatous polyposis (FAP) are decreased by the administration of nonsteroidal anti-inflammatory drugs [2]. COX is a key enzyme in the conversion of arachidonic acid to prostaglandin and exists as two isoforms, COX-1 and COX-2 [3]. COX-2 is a highly inducible gene, activated by cytokines, growth factors, phorbol esters, oncogenes and chemical carcinogens [3]. The overexpression of COX-2 has been reported in a wide variety of malignant human tumours. Thus, the availability of COX-2 inhibitors offers the potential for targeted cancer therapy or chemoprevention, with celecoxib already shown to significantly reduce the number of colorectal polyps in patients with FAP [4].

In NSCLC, an increase in COX-2 expression has been detected in adenocarcinomas (ADC) and squamous cell carcinomas (SQCC) [5-7] in comparison with normal lung tissue; however, higher levels were observed in ADC relative to SQCC. In addition, COX-2 expression was shown to be a negative prognostic factor for the survival of patients with NSCLC $[8,9]$. COX-2 expression was also found to be increased in atypical adenomatous hyperplasia, a possible precursor of $\operatorname{ADC}[6,7,10,11]$, but to the current authors' knowledge, it has never been studied in preneoplastic lesions presumed to precede SQCC. COX-2 inhibitors are currently being tested in
AFFILIATIONS

*Dept of Intensive Care and Thoracic Oncology,

\#Dept of Pathology, Institut Jules Bordet, and

'Dept of Pneumology, CHU SaintPierre, Brussels, Belgium.

CORRESPONDENCE

C. Mascaux

Institut Jules Bordet rue Héger-Bordet, 1 B-1000 Brussels

Belgium

Fax: 3225343756

E-mail: celine.mascaux@bordet.be

Received:

January 052005

Accepted after revision:

May 032005

SUPPORT STATEMENT

C. Mascaux was supported by a fellowship from the National Fund for Scientific Research (FNRS, Belgium; 1. 1. 148.04.F) and is a FNRS Research Fellow. This study was also supported by a grant from the FNRS (FRSM; 3.4624.04) and a grant from the ASBL Les Amis de I'Institut Jules Bordet. 
ongoing phase II studies for the chemoprevention of NSCLC, based on pre-clinical studies in mice $[12,13]$. In the present study, COX-2 expression was examined in all types of bronchial precursor lesions from SQCC using immunohistochemistry (IHC) to determine whether COX-2 expression increases during SQCC carcinogenesis and, if so, when it occurs.

\section{MATERIALS AND METHODS Study population}

Eligible patients had a minimum smoking exposure of 30 packyrs and/or a history of lung or head and neck cancer. Laserinduced fluorescence bronchoscopy was performed under local anaesthetic, and any area that appeared as abnormal by fluorescence was biopsied [14]. Only 14 in situ carcinoma (CIS) were obtained, which was the rarest precursor in the cohort, leading to a parallel analysis of 14 samples of each histological type (normal $(\mathrm{Nl})$, hyperplasia $(\mathrm{H})$, metaplasia $(\mathrm{M})$, mild dysplasia (MiD), moderate dysplasia (MoD), severe dysplasia (SD), and CIS). Eight biopsies from invasive carcinoma (IC) that was diagnosed during fluorescence bronchoscopy were also analysed. In total, 106 consecutively eligible biopsies from 106 different patients, collected between February 1996 and November 2002 were reviewed. Only the most severe lesion found in an individual patient was evaluated and each patient was included in the study only once. All 106 eligible patients provided sufficiently large biopsies for an evaluation of COX-2 expression. There were 19 females and 87 males with a median age of 63 yrs (range: 38-92 yrs). A total of 42 patients were current smokers, 62 former smokers, and for two, their smoking habits were unknown. The patients had smoked a median of 50 pack-yrs of tobacco (range 19-134 yrs). The bronchoscopic procedure was performed on 18 patients for lung cancer detection, 19 as pre-operative work-up for bronchial (10) or head and neck (9) cancer, 45 for follow-up of a previously treated lung cancer, 22 for follow-up of CIS and two for other reasons.

\section{Sample preparation and selection}

All biopsies were routinely fixed for $3 \mathrm{~h}$ (minimum) to $10 \mathrm{~h}$ (maximum), in 10\% neutral buffered formalin immediately following the bronchoscopy and subsequently embedded in paraffin. From each paraffin-embedded tissue blocks, $4 \mu \mathrm{m}$ sections were cut and deposited on SuperFrost Plus Slides (Menzel-Gläser, Braunschwein, Germany). All of the haematoxylin- and eosin- stained lesions were classified by a pathologist using the 1999 histological World Health Organization (WHO)/International Association for the Study of Lung Cancer (IASLC) classification [15] into: Nl, H, M, MiD, $\mathrm{MoD}, \mathrm{SD}, \mathrm{CIS}$ and IC.

\section{Immunohistochemistry for COX-2}

All reagents were of analytical quality and did not require further purification. Methanol, citric acid, sodium citrate, tris(hydroxymethyl)aminomethane (TRIS) and chloridric acid were purchased from Merck (Darmstad, Germany).

IHC was performed using a standard avidin-biotin-peroxydase complex [16] and a mouse monoclonal antibody directed against the human COX-2 sequence (amino acids 580-599; immunoglobulin (Ig)-G1) from the Cayman Chemical Company (Ann Arbor, USA), which stains the cytoplasm.
The paraffin was removed from the slides by xylene and the tissue was rehydrated in ethanol. Endogenous peroxydase activity was quenched by incubating the slides in $0.3 \%$ hydrogen peroxide in methanol for $30 \mathrm{~min}$ at room temperature followed by two washes in $0.05 \mathrm{M}$ TRIS-HCl pH 7.6 in $0.9 \% \mathrm{NaCl}$ for $10 \mathrm{~min}$. Antigen retrieval was accomplished in $0.01 \mathrm{M}$ citrate buffer $\mathrm{pH} 6$ coupled with $15 \mathrm{~min}$ of microwave heating at $650 \mathrm{~W}$, followed by $25 \mathrm{~min}$ of cooling at room temperature. The anti-COX-2 antibody (dilution 1:24; final titration $4.17 \mathrm{ng} \cdot \mathrm{\mu L}^{-1}$ ) was incubated on the slide for $60 \mathrm{~min}$ at $37^{\circ} \mathrm{C}$. The following steps were performed automatically at $37^{\circ} \mathrm{C}$ using the NexES system (Ventana Medical Systems, Tucson, AZ, USA). The primary antigen-antibody complex was fixed using $0.05 \%$ glutaraldehyde in $0.9 \% \mathrm{NaCl}$ followed by incubation with a secondary biotinylated antibody for $8 \mathrm{~min}$. The slides were stained using a diaminobenzidine detection kit (Ventana Medical Systems) and counterstained with haematoxylin.

Negative controls included omitting the primary antibody and its substitution with a normal mouse IgG. COX-2-positive colon cancers, which were also positive on the slide in the normal smooth muscle, and known COX-2 positive NSCLC were used as positive controls.

\section{Interpretation of immunohistochemical staining}

Two independent assessments were performed by an oncologist C. Mascaux and a biologist B. Martin, both of whom are trained in the IHC examination of bronchial epithelial samples. Each independently evaluated the results of the IHC staining twice. The observations of these two evaluators were compared and discordant interpretations were resolved by a common review of the slides in question using a multi-head microscope. All analyses were performed blind to patient background information.

Only cytoplasmic staining was examined for the assessment of COX-2 expression. The distribution of COX-2 was, thus, scored as: 1) no staining $(0 \%) ; 2)$ foca1 staining $(1-49 \%)$; and 3$)$ diffuse staining ( $\geqslant 50 \%$ of the cells). The staining intensity was defined as: $0=$ no staining; weak staining $=$ intensity inferior or equal to those of the normal epithelial cells in the lung; moderate staining=stronger intensity than that of normal lung epithelial cells, such as some NSCLC, colon cancer cells or smooth muscle in colon; and strong staining=very intense staining, stronger than that of colon smooth muscle or endothelial cells, equal to the strongest staining seen in NSCLC cells or colic cancer cells.

\section{Statistical assessment}

Comparison between the histological groups and distribution of positive or negative biopsies for COX-2 expression were performed using the Chi-squared test and bilateral Fisher's test. The level of significance was set at $p \leqslant 0.05$.

\section{RESULTS}

To check the quality of the authors' external control, COX-2 immunostaining of colon cancer specimens was assessed, both in the cancer cells as well as the smooth muscle cells and of NSCLC samples that included normal lung tissue on the same slide. COX-2 staining was found only in the cytoplasm. In colon cancer, three patterns of intensity were detected: 
1) weakly positive cells among the normal glandular colic cells; 2) moderately positive cells located in the smooth muscle, vascular endothelium or among some cancer cells; and 3) intensely positive cells corresponding to the large majority of cancer cells. COX-2 staining in NSCLC with normal lung tissue on the same slides also showed three patterns of staining. Normal epithelial bronchial cells had diffuse, but weak staining, whereas positive NSCLC were stained with moderate or strong intensity similar to the colon cancer slides. In preneoplastic lesions, using the same criteria as for colon and $\mathrm{NSClC}$, three different staining intensities were observed: weak, moderate or strong. The initial inspection of staining in bronchial pre-neoplastic lesions revealed three distinct distributions of positive cells: all cells were negative or a minority (focal staining) or a majority of cells (diffuse staining) were stained. Staining of bronchial lesions was scored as follows (fig. 1): $0=$ no staining or staining of the basal cell layer only; $1+$ positive $=$ focal or diffuse weak staining $(0-100 \%$ of positive cells with weak staining); $2+$ positive $=$ focal moderate or strong staining $(<50 \%$ of positive cells with moderate or strong

\begin{tabular}{lcc} 
TABLE 1 & $\begin{array}{c}\text { Scoring of the immunohistochemical staining } \\
\text { (1-49\% of } \\
\text { stained cells }\end{array}$ & $\begin{array}{c}\mathbf{5 0 - 1 0 0 \%} \text { of } \\
\text { stained cells }\end{array}$ \\
\hline Weak intensity & $1+$ & $1+$ \\
Moderate intensity & $2+$ & $3+$ \\
Strong intensity & $2+$ & $3+$ \\
\hline
\end{tabular}

staining); and $3+$ positive $=$ diffuse moderate or strong staining ( $>50 \%$ of positive cells with moderate or strong staining; table 1). Any precursor lesion showing more staining than normal lung tissue was considered to be positive. Lesions that were 0 or $1+$ positive were considered as negative (no increase in COX-2 expression compared with normal lung tissue) and those that were $2+$ or $3+$ positive were considered as positive (an increase in COX-2 expression compared with normal lung tissue).
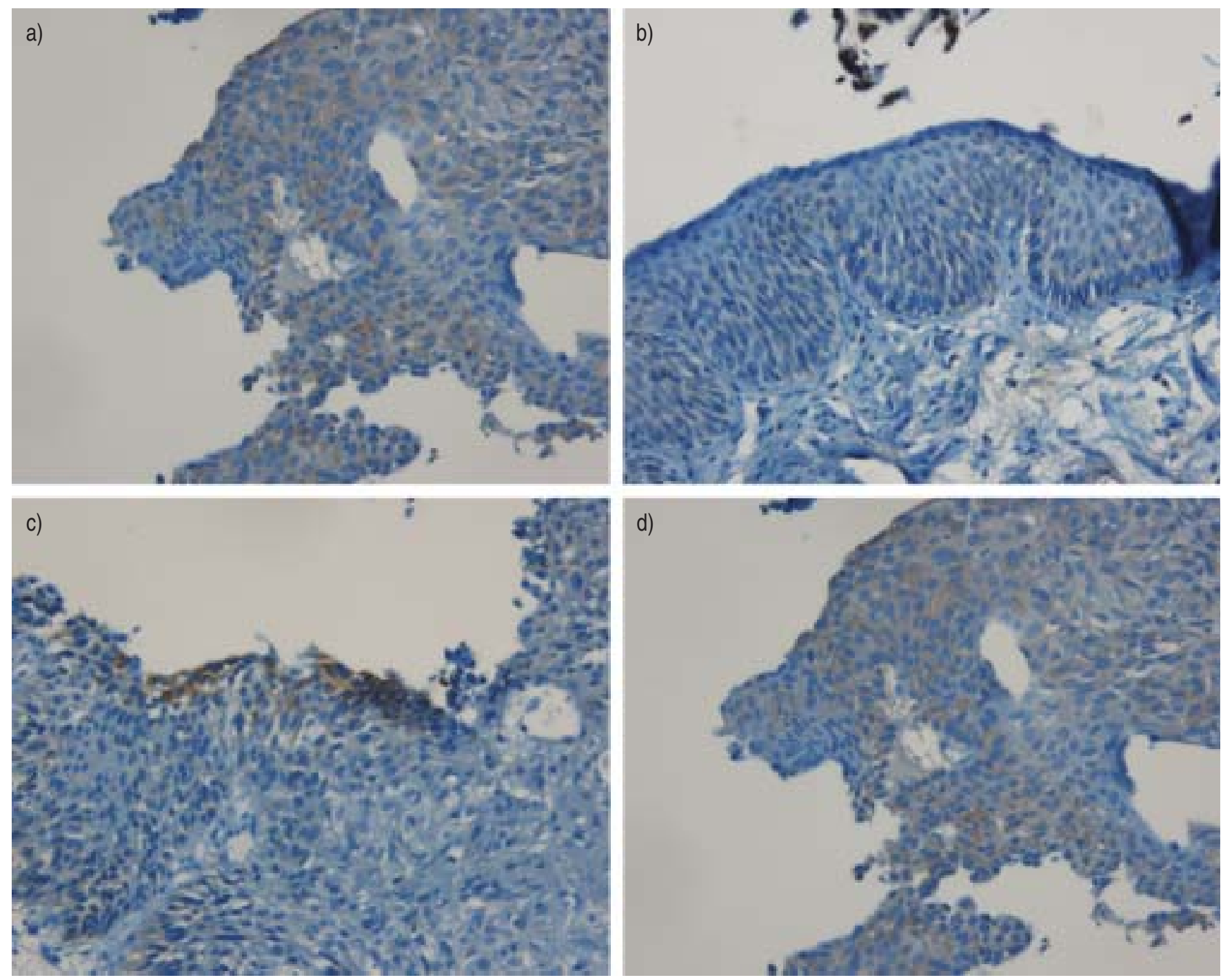

FIGURE 1. Immunohistochemical staining scores showing a) 0 : no staining, b) 1+ positive: diffuse weak staining, c) 2+ positive: focal strong staining, and d) $3+$ positive: diffuse moderate staining 


\begin{tabular}{|c|c|c|c|c|c|c|}
\hline \multirow[t]{3}{*}{ TABLE 2} & $\begin{array}{l}\text { ooxyg } \\
\text { tage }\end{array}$ & $\begin{array}{l}\text { nase } \\
\text { the } p\end{array}$ & $\begin{array}{l}\text { X)-2 e } \\
\text { neopla }\end{array}$ & $\begin{array}{l}\text { xpress } \\
\text { stic le }\end{array}$ & $\begin{array}{l}\text { on acc } \\
\text { ion }\end{array}$ & ling to \\
\hline & \multirow[t]{2}{*}{ Total } & \multicolumn{2}{|c|}{ cox-2 negative } & \multirow[t]{2}{*}{ Total } & \multicolumn{2}{|c|}{ COX-2 positive } \\
\hline & & $0+$ & $1+$ & & $2+$ & $3+$ \\
\hline Normal & 14 & 13 & 1 & 0 & 0 & 0 \\
\hline Hyperplasia & 14 & 13 & 1 & 0 & 0 & 0 \\
\hline Metaplasia & 14 & 12 & 2 & 0 & 0 & 0 \\
\hline Mild dysplasia & 14 & 13 & 1 & 0 & 0 & 0 \\
\hline $\begin{array}{l}\text { Moderate } \\
\text { dysplasia }\end{array}$ & 14 & 10 & 4 & 0 & 0 & 0 \\
\hline Severe dysplasia & 6 & 4 & 2 & 8 & 8 & 0 \\
\hline In situ carcinoma & 6 & 4 & 2 & 8 & 6 & 2 \\
\hline Invasive carcinoma & 3 & 3 & 0 & 5 & 4 & 1 \\
\hline Overall & 85 & 72 & 13 & 21 & 18 & 3 \\
\hline \multicolumn{7}{|c|}{$\begin{array}{l}\text { Data are presented as } n \text {. Negative: no staining or weak staining; positive: } \\
\text { moderate or strong staining whatever the focal or diffuse distribution; } 0+: \text { no } \\
\text { staining; } 1+\text { : focal or diffuse weak staining; } 2+\text { f focal moderate or strong } \\
\text { staining; } 3+\text { : diffuse moderate or strong staining. }\end{array}$} \\
\hline
\end{tabular}

The current authors found that 21 biopsies were positive $(20 \%)$ and 85 were negative $(80 \%)$ for COX-2 expression. The distribution of COX-2 expression based on lesion stage is shown in table 2. Positive biopsies were detected in $57.14 \%$ of SD, $57.14 \%$ of CIS and $62.5 \%$ of IC. Thus, $58.33 \%$ of the high-grade biopsies (defined as lesions-corresponding to severe dysplasia or worse) were positive for COX-2. In contrast, all 70 lesions classified as NI, H, M, MiD, MoD (low-grade) were negative for COX-2 staining, 61 (87\%) were graded 0 and nine (13\%) $1+$ positive. Among all 21 positive biopsies, 18 were $2+$ or had focal staining (eight SD, six CIS and four IC) and three were 3+ or had diffuse staining (two CIS and one IC). It was also observed that in biopsies with focal staining, cells with more atypia were those expressing COX-2.

The positive slides were also assessed for COX-2 expression in both their normal tissue and low-grade lesions adjacent to high-grade lesions. This analysis revealed that COX-2 expression was increased only in the high-grade lesions from these patients. Of the 21 positive slides, 10 contained both normal and low-grade lesions with high-grade lesions, including some with several areas of normal tissue or lowgrade lesions in the same biopsy. A total of five normal tissues, eight hyperplasia and three metaplasia were found in the positive biopsies, with all 16 of these lesions being negative for COX-2 expression.

A statistically significant difference in COX-2 expression was found between $\mathrm{Nl}, \mathrm{H}, \mathrm{M}, \mathrm{MiD}, \mathrm{MoD}$ as a group and $\mathrm{SD}, \mathrm{CIS}$ and IC as a separate group $(\mathrm{p}<0.001)$. Grouping MiD and MoD versus SD $(\mathrm{p}<0.001)$ or $\mathrm{MiD}$ and $\mathrm{MoD}$ versus $\mathrm{SD}$ and $\mathrm{CIS}$ $(p<0.001)$ also revealed statistically significant differences. COX-2 expression did not discriminate SD and CIS from IC $(p=0.10)$. The positive predictive value for high-grade bronchial pre-malignant lesions (SD, CIS, IC) when COX-2 expression is increased was $100 \%$. The negative predictive value was $82.35 \%$.

\section{DISCUSSION}

The present study demonstrates that COX-2 expression is increased in precursors of SQCC, but only in high-grade bronchial pre-neoplastic lesions (SD, CIS) and early invasive occult SQCC. SD appears to be the cut-off for COX-2 expression. The current authors' data extend previous studies of pre-neoplastic lesions [17-19], which also showed that SD may be the key step in early lung carcinogenesis, separating low-grade lesions from high-grade lesions that are more prone to progress to invasive cancer. In routine clinical practice, the classification of pre-malignant lesions into low versus highgrade stages could provide a fairer representation of their clinical behaviour than the 1999 WHO/IASCL classification system. However, this hypothesis requires further investigation for confirmation. Moreover, positive COX-2 expression was detected only in the high-grade lesions from patients that had both positive high-grade lesions and adjacent normal tissue or low-grade lesions. These data argue for no field effect of COX-2 expression, but only for a grade effect. The present study is the first to assess COX-2 expression in biopsies from all precursors of SQCC, with little previous comparable data available. KIM et al. [20] reported that a few hyperplastic and non-neoplastic bronchial epithelial cells adjacent to the tumour had very weak COX-2 expression. HASTÜRK et al. [11], analysed 51 hyperplasia and 15 metaplasia and found they were all negative for COX-2 expression, which is in agreement with the present results. The first and only previous data concerning COX-2 expression in dysplasia and CIS appeared in the study by HiDA et al. [6], where COX-2 expression was assessed in four metaplasia, four dysplasia and four CIS. None of their lesions exhibited increased expression of COX-2 [6]. For dysplasia, the increase in COX-2 expression observed was not comparable with this previous study, because HiDA et al. [6] did not use the new 1999 WHO/IASCL classification nor distinguish MiD from MoD or SD. In contrast, the four CIS assessed by HIDA et al. [6] did not display increased COX-2 expression, whereas five out of eight CIS were positive for COX-2 expression in the current study. This discrepancy could be explained by both the small number of samples studied by HIDA et al. [6] and the different thresholds chosen to define COX-2 overexpression.

Among biopsies with increased COX-2 expression, two different subtypes were distinguished; one with focal staining (18 out of 21 positive lesions or $85.71 \%$ ) and another with diffuse staining (three out of 21). In the focal group, cells expressing COX-2 strongly could behave differently from other cells in the same lesion. The role of COX-2 in oncogenesis has been widely studied in vitro and in vivo using animal models. COX-2 can interfere with most pathways implicated in lung carcinogenesis, such as microvascular angiogenesis [21], resistance to apoptosis [22, 23], host immunity [24] and cell adhesion [25]. Moreover, COX-2 appears to be a prognostic factor in NSCLC, particularly at the earliest stages $[8,9]$. Thus, it is possible that among bronchial pre-neoplastic lesions, COX2 expressing cells could be those initiating the progression to malignancy. The current observation that cells with increased COX-2 expression are those showing more atypia favour this hypothesis.

Although COX-2 is induced by inflammation and oncogenic stimuli, it can also be decreased by other pathological 
mechanisms. A recent study found that COX-2 expression is reduced in interstitial diseases such as idiopathic pulmonary fibrosis and sarcoidosis in comparison with normal lung tissue [26].

COX-2 should be considered a potentially interesting marker for the early detection of patients with a high risk of lung cancer. In the present study, the positive predictive value of COX-2 expression for predicting the detection of a highgrade pre-neoplastic lesion (SD, CIS or CI) was $100 \%$. The negative predictive value of an absence of COX-2 overexpression was $82.35 \%$ for the absence of a high-grade lesion. COX-2 expression, thus, seems to be an interesting potential marker for the high risk of developing NSCLC. It could be further speculated that high-grade lesions that do not express COX-2 might have a better prognosis than those that do. However, a descriptive analysis cannot show any addictive value for the prediction of lung cancer risk in a high risk population compared with the histological grade itself. A prospective study should demonstrate whether there is added predictive value from COX-2 expression in high-grade lesions.

Based on the present results, showing COX-2 expression in high-grade lesions, COX-2 appears to be a potentially promising target for chemoprevention of lung cancer. The goals of chemopreventive therapy are 2-fold; to promote regression of the more aggressive cells that already express COX-2, and to prohibit the progression of the other cells that do not yet express COX-2. COX-2 inhibitors also possess different COX-2 independent anti-cancer properties [27, 28], and several ongoing trials are underway to assess the role of COX-2 inhibitors in chemoprevention of NSCLC. These trials are based on data acquired in pre-clinical animal model studies [12, 13, 29-32]. However, clinical data were still missing. The current study provides more information concerning COX-2 in SQCC oncogenesesis, and suggests there may be a potentially promising role for COX-2 inhibitors for the chemoprevention of lung cancer. The risk from these drugs in relationship to the recent discovery of long-term cardiotoxicity by some COX-2 inhibitors should be balanced with the potential advantages before considering these drugs in clinical practice for chemoprevention, which necessitates their use for years.

In conclusion, cyclooxygenase-2, an enzyme that plays a key role in several lung carcinogenesis pathways, is increased during early lung carcinogenesis of squamous cell carcinoma and segregates low-grade lesions from high-grade pre-malignant lesions. Cyclooxygenase- 2 appears to be a potential early marker of lung malignancy with a high positive predictive value, although prospective studies are still required to demonstrate the potential additive value to histological grade. Cyclooxygenase-2 should be considered as a good intermediate-point marker if chemoprevention based on cyclooxygenase- 2 inhibition is performed in future clinical trials.

\section{ACKNOWLEDGMENTS}

The authors would like to thank K. Willard-Gallo for critical reading of the manuscript.

\section{REFERENCES}

1 Greenlee RT, Murray T, Bolden S, Wingo PA. Cancer statistics, 2000. CA Cancer J Clin 2000; 50: 7-33.

2 Waddell WR, Loughry RW. Sulindac for polyposis of the colon. J Surg Oncol 1983; 24: 83-87.

3 Smith WL, Langenbach R. Why there are two cyclooxygenase isozymes. J Clin Invest 2001; 107: 1491-1495.

4 Steinbach G, Lynch PM, Phillips RK, et al. The effect of celecoxib, a cyclooxygenase-2 inhibitor, in familial adenomatous polyposis. N Engl J Med 2000; 342: 1946-1952.

5 Ochiai M, Oguri T, Isobe T, Ishioka S, Yamakido M. Cyclooxygenase-2 (COX-2) mRNA expression levels in normal lung tissues and non-small cell lung cancers. Jpn J Cancer Res 1999; 90: 1338-1343.

6 Hida T, Yatabe Y, Achiwa H, et al. Increased expression of cyclooxygenase 2 occurs frequently in human lung cancers, specifically in adenocarcinomas. Cancer Res 1998; 58: 3761-3764.

7 Wolff H, Saukkonen K, Anttila S, Karjalainen A, Vainio H, Ristimaki A. Expression of cyclooxygenase-2 in human lung carcinoma. Cancer Res 1998; 58: 4997-5001.

8 Khuri FR, Wu H, Lee JJ, et al. Cyclooxygenase-2 overexpression is a marker of poor prognosis in stage I nonsmall cell lung cancer. Clin Cancer Res 2001; 7: 861-867.

9 Achiwa H, Yatabe Y, Hida T, et al. Prognostic significance of elevated cyclooxygenase 2 expression in primary, resected lung adenocarcinomas. Clin Cancer Res 1999; 5: 1001-1005.

10 Hosomi Y, Yokose T, Hirose Y, et al. Increased cyclooxygenase 2 (COX-2) expression occurs frequently in precursor lesions of human adenocarcinoma of the lung. Lung Cancer 2000; 30: 73-81.

11 Hasturk S, Kemp B, Kalapurakal SK, Kurie JM, Hong WK, Lee JS. Expression of cyclooxygenase-1 and cyclooxygenase- 2 in bronchial epithelium and nonsmall cell lung carcinoma. Cancer 2002; 94: 1023-1031.

12 Wardlaw SA, March TH, Belinsky SA. Cyclooxygenase-2 expression is abundant in alveolar type II cells in lung cancer-sensitive mouse strains and in premalignant lesions. Carcinogenesis 2000; 21: 1371-1377.

13 Rioux N, Castonguay A. Prevention of NNK-induced lung tumorigenesis in A/J mice by acetylsalicylic acid and NS398. Cancer Res 1998; 58: 5354-5360.

14 Vermylen P, Pierard P, Roufosse C, et al. Detection of bronchial preneoplastic lesions and early lung cancer with fluorescence bronchoscopy: a study about its ambulatory feasibility under local anaesthesis. Lung Cancer 1999; 25: 161-168.

15 Travis WD, Colby TV, Shimosato Y, et al. WHO/IASCL Histological classification of tumours. Histological typing of Lung and Pleural tumors. 3rd Edn. New York, Springer Verlag, 1999.

16 King G, Payne S, Walker F, Murray GI. A highly sensitive detection method for immunohistochemistry using biotinylated tyramine. J Pathol 1997; 183: 237-241.

17 Meert AP, Feoli F, Martin B, et al. Ki67 expression in bronchial preneoplastic lesions and carcinoma in situ defined according to the new 1999 WHO/IASLC criteria: a preliminary study. Histopathology 2004; 44: 47-53.

18 Meert AP, Verdebout JM, Martin B, Ninane V, Feoli F, Sculier JP. Epidermal growth factor receptor expression in 
pre-invasive and early invasive bronchial lesions. Eur Respir J 2003; 21: 611-615.

19 Jeanmart M, Lantuejoul S, Fievet F, et al. Value of immunohistochemical markers in preinvasive bronchial lesions in risk assessment of lung cancer. Clin Cancer Res 2003; 9: 2195-2203.

20 Kim HS, Youm HR, Lee JS, Min KW, Chung JH, Park CS. Correlation between cyclooxygenase-2 and tumor angiogenesis in non-small cell lung cancer. Lung Cancer 2003; 42: 163-170.

21 Masferrer JL, Leahy KM, Koki AT, et al. Antiangiogenic and antitumor activities of cyclooxygenase-2 inhibitors. Cancer Res 2000; 60: 1306-1311.

22 Hida T, Kozaki K, Muramatsu H, et al. Cyclooxygenase-2 inhibitor induces apoptosis and enhances cytotoxicity of various anticancer agents in non-small cell lung cancer cell lines. Clin Cancer Res 2000; 6: 2006-2011.

23 Liu XH, Yao S, Kirschenbaum A, Levine AC. NS398, a selective cyclooxygenase-2 inhibitor, induces apoptosis and down-regulates bcl-2 expression in $\mathrm{LNCaP}$ cells. Cancer Res 1998; 58: 4245-4249.

24 Huang M, Stolina M, Sharma S, et al. Non-small cell lung cancer cyclooxygenase-2-dependent regulation of cytokine balance in lymphocytes and macrophages: upregulation of interleukin 10 and down-regulation of interleukin 12 production. Cancer Res 1998; 58: 1208-1216.

25 Tsujii M, Kawano S, Dubois RN. Cyclooxygenase-2 expression in human colon cancer cells increases metastatic potential. Proc Natl Acad Sci USA 1997; 94 3336-3340.

26 Petkova DK, Clelland CA, Ronan JE, Lewis S, Knox AJ. Reduced expression of cyclooxygenase (COX) in idiopathic pulmonary fibrosis and sarcoidosis. Histopathology 2003; 43: 381-386.

27 Hwang DH, Fung V, Dannenberg AJ. National Cancer Institute workshop on chemopreventive properties of nonsteroidal anti-inflammatory drugs: role of COX-dependent and -independent mechanisms. Neoplasia 2002; 4: 91-97.

28 Patel MI, Subbaramaiah K, Du B, et al. Celecoxib inhibits prostate cancer growth: evidence of a cyclooxygenase-2independent mechanism. Clin Cancer Res 2005; 11: 1999-2007.

29 Wiese FW, Thompson PA, Kadlubar FF. Carcinogen substrate specificity of human COX-1 and COX-2. Carcinogenesis 2001; 22: 5-10.

30 Eling TE, Thompson DC, Foureman GL, Curtis JF, Hughes MF. Prostaglandin $\mathrm{H}$ synthase and xenobiotic oxidation. Annu Rev Pharmacol Toxicol 1990; 30: 1-45.

31 Ho IC, Lee TC. Arsenite pretreatment attenuates benzo[a]pyrene cytotoxicity in a human lung adenocarcinoma cell line by decreasing cyclooxygenase-2 levels. J Toxicol Environ Health A 2002; 65: 245-263.

32 Kelley DJ, Mestre JR, Subbaramaiah K, et al. Benzo[a]pyrene up-regulates cyclooxygenase-2 gene expression in oral epithelial cells. Carcinogenesis 1997; 18: 795-799. 